\title{
SOME METRIC PROPERTIES OF THE TEICHMÜLLER SPACE OF A CLOSED SET IN THE RIEMANN SPHERE
}

\author{
Nishan Chatterjee \\ Graduate Center of the City University of New York, Department of Mathematics \\ 365 Fifth Avenue, New York, NY 10016, U.S.A.; nchatterjee@gradcenter.cuny.edu
}

\begin{abstract}
Let $E$ be an infinite closed set in the Riemann sphere, and let $T(E)$ denote its Teichmüller space. In this paper, we study some metric properties of $T(E)$. We prove Earle's form of Teichmüller contraction for $T(E)$, holomorphic isometries from the open unit disk into $T(E)$, extend Earle's form of Schwarz's lemma for classical Teichmüller spaces to $T(E)$, and finally study complex geodesics and unique extremality for $T(E)$.
\end{abstract}

\section{Introduction}

Let $\mathbf{C}$ denote the complex plane, $\Delta:=\{z \in \mathbf{C}:|z|<1\}$ denote the open unit disk and $\widehat{\mathbf{C}}=\mathbf{C} \cup\{\infty\}$ denote the Riemann sphere. Throughout this paper, we will assume that $E$ is a closed set in $\widehat{\mathbf{C}}$ and that 0,1 , and $\infty$ belong to $E$. The Teichmüller space of $E$, denoted by $T(E)$, was first studied by Lieb in his 1990 Cornell University dissertation [14], written under the direction of Earle. It has several applications in holomorphic motions, geometric function theory, and holomorphic families of Möbius groups; see the papers $[7,12,15,18]$. In this paper, we study some metric properties of $T(E)$. Our paper is arranged as follows. In $\S 1$, we give the relevant definitions and also state various properties of $T(E)$ that will be necessary in our paper. In $\S 2$, we state the main theorems of our paper and also the motivations for these results. In $\S \S 3-6$, we give the proofs of our main theorems.

\section{Teichmüller space of a closed set in $\widehat{\mathrm{C}}$}

We call a homeomorphism of $\widehat{\mathbf{C}}$ normalized if it fixes the points 0,1 , and $\infty$. Let $M(\mathbf{C})$ denote the open unit ball of the complex Banach space $L^{\infty}(\mathbf{C})$. For each $\mu$ in $M(\mathbf{C})$, there exists a unique normalized quasiconformal homeomorphism of $\widehat{\mathbf{C}}$ onto itself that has Beltrami coefficient $\mu$, denoted by $w^{\mu}$.

Definition 1.1. The normalized quasiconformal self-mappings $f$ and $g$ of $\widehat{\mathbf{C}}$ are said to be $E$-equivalent if and only if $f^{-1} \circ g$ is isotopic to the identity rel $E$. The Teichmüller space $T(E)$ is the set of all $E$-equivalence classes of normalized quasiconformal self-mappings of $\widehat{\mathbf{C}}$. The basepoint of $T(E)$ is the E-equivalence class of the identity map.

We define the projection

$$
P_{E}: M(\mathbf{C}) \rightarrow T(E)
$$

https://doi.org/10.5186/aasfm.2017.4247

2010 Mathematics Subject Classification: Primary 32G15, 30F60; Secondary 32H02.

Key words: Teichmüller space of a closed set, Teichmüller contraction, holomorphic isometries, Schwarz's lemma, complex geodesics. 
by setting $P_{E}(\mu)$ equal to the $E$-equivalence class of $w^{\mu}$, written as $\left[w^{\mu}\right]_{E}$. Clearly, $P_{E}$ maps the basepoint of $M(\mathbf{C})$ to the basepoint of $T(E)$. (We will use the same notation 0 for the basepoints in $M(\mathbf{C})$ and $T(E)$.)

In his doctoral dissertation [14], Lieb proved that $T(E)$ is a complex Banach manifold such that the projection map $P_{E}$ is a holomorphic split submersion. For more details, see [7].

The tangent space at the basepoint. Let $A(E)$ be the closed subspace of $L^{1}(\mathbf{C})$ consisting of the functions $f$ in $L^{1}(\mathbf{C})$ whose restriction to $E^{c}$ is holomorphic. We identify $L^{\infty}(\mathbf{C})$ with the dual space of $L^{1}(\mathbf{C})$ in the usual way. Set

$$
A(E)^{\perp}=\left\{\mu \in L^{\infty}(\mathbf{C}): \ell_{\mu}(f)=\iint_{\mathbf{C}} \mu(z) f(z) d x d y=0 \text { for all } f \text { in } A(E)\right\} .
$$

Proposition 1.2. (Teichmüller's lemma for $T(E)) \operatorname{ker}\left(P_{E}^{\prime}(0)\right)=A(E)^{\perp}$.

See Proposition 7.18 in [7].

Corollary 1.3. The tangent space to $T(E)$ at its basepoint is naturally isomorphic to $A(E)^{*}$, the dual space of $A(E)$.

The natural isomorphism sends the tangent vector $P_{E}^{\prime}(0) \mu$ to the linear functional $f \mapsto \ell_{\mu}(f)$ on $A(E)$.

Changing the basepoint. Let $h$ be a normalized quasiconformal self-mapping of $\widehat{\mathbf{C}}$, and let $\widetilde{E}=h(E)$. By definition, the allowable map $h^{*}$ from $T(\widetilde{E})$ to $T(E)$ maps the $\widetilde{E}$-equivalence class of $g$ to the $E$-equivalence class of $g \circ h$ for every normalized quasiconformal self-mapping $g$ of $\widehat{\mathbf{C}}$.

Proposition 1.4. The allowable map $h^{*}: T(\widetilde{E}) \rightarrow T(E)$ is biholomorphic. If $\mu$ is the Beltrami coefficient of $h$, then $h^{*}$ maps the basepoint of $T(\widetilde{E})$ to the point $P_{E}(\mu)$ in $T(E)$.

Forgetful maps. If $E$ is a subset of the closed set $\widetilde{E}$ and $\mu$ is in $M(\mathbf{C})$, then the $\widetilde{E}$-equivalence class of $w^{\mu}$ is contained in the $E$-equivalence class of $w^{\mu}$. Therefore, there is a well-defined forgetful map $p_{\widetilde{E}, E}$ from $T(\widetilde{E})$ to $T(E)$ such that $P_{E}=p_{\widetilde{E}, E} \circ P_{\widetilde{E}}$.

Proposition 1.5. The forgetful map $p_{\widetilde{E}, E}$ is a basepoint preserving holomorphic split submersion.

Proof. Since $P_{E}=p_{\widetilde{E}, E} \circ P_{\widetilde{E}}$ and $P_{E}$ and $P_{\widetilde{E}}$ are holomorphic split submersions, so is $p_{\widetilde{E}, E}$.

The following proposition will be very useful in our paper.

Proposition 1.6. Let $f$ be any holomorphic map of $\Delta$ into $T(E)$ and let $\mu$ be any point in $M(\mathbf{C})$ such that $P_{E}(\mu)=f(0)$. There is a holomorphic map $\widehat{f}$ from $\Delta$ to $M(\mathbf{C})$ such that $\widehat{f}(0)=\mu$ and $P_{E} \circ \widehat{f}=f$.

For proofs see Proposition 7.27 in [7] or Proposition 5.1 in [17]. This is an easy consequence of the "universal" property of $T(E)$ (see [15]) and Slodkowski's theorem on extensions of holomorphic motions (see [21]).

The Kobayashi and Teichmüller metrics on $T(E)$.

Proposition 1.7. The Kobayashi metric on $M(\mathbf{C})$ is given by

$$
\rho_{M}(\mu, \nu)=\tanh ^{-1}\left\|\frac{(\mu-\nu)}{(1-\bar{\mu} \nu)}\right\|_{\infty}
$$


for all $\mu, \nu$ in $M(\mathbf{C})$. The infinitesimal Kobayashi metric on $M(\mathbf{C})$ is given by

$$
K_{M}(\mu, \lambda)=\left\|\frac{\lambda}{\left(1-|\mu|^{2}\right)}\right\|_{\infty}
$$

for $\mu$ in $M(\mathbf{C})$ and $\lambda$ in $L^{\infty}(\mathbf{C})$.

See Proposition 7.25 in [7].

By definition, the Teichmüller metric $d_{T(E)}$ on $T(E)$ is given by

$$
d_{T(E)}\left(P_{E}(\mu), t\right)=\inf \left\{\rho_{M}(\mu, \nu): \nu \in M(\mathbf{C}) \text { and } P_{E}(\nu)=t\right\}
$$

for all $\mu$ in $M(\mathbf{C})$ and $t$ in $T(E)$.

The infinitesimal Teichmüller metric $F_{T(E)}$ is defined on the tangent bundle of $T(E)$ by the formula

$$
F_{T(E)}\left(P_{E}(\mu), v\right)=\inf \left\{K_{M}(\mu, \lambda): \lambda \in L^{\infty}(\mathbf{C}) \text { and } P_{E}^{\prime}(\mu) \lambda=v\right\},
$$

for any $\mu$ in $M(\mathbf{C})$ and tangent vector $v$ to $T(E)$ at the point $P_{E}(\mu)$.

Proposition 1.8. The Teichmüller and Kobayashi metrics on $T(E)$ are equal, and the infinitesimal Teichmüller and Kobayashi metrics are also equal.

See Proposition 7.30 in [7].

Definition 1.9. A map $f: \Delta \rightarrow T(E)$ is called a holomorphic isometry if $f$ is holomorphic and for any pair $t, t^{\prime}$ in $\Delta, d_{T(E)}\left(f(t), f\left(t^{\prime}\right)\right)=\rho_{\Delta}\left(t, t^{\prime}\right)$.

Recall that the Poincaré metric on $\Delta$ is given by

$$
\rho_{\Delta}(z, w)=\tanh ^{-1}\left|\frac{z-w}{1-\bar{z} w}\right|
$$

for all $z$ and $w$ in $\Delta$.

Definition 1.10. A Beltrami coefficient $\mu$ in $M(\mathbf{C})$ is called extremal in its $E$ equivalence class, if $P_{E}(\mu)=P_{E}(\nu)$ and $\|\mu\|_{\infty} \leq\|\nu\|_{\infty}$. Equivalently, $\mu$ in $M(\mathbf{C})$ is extremal in its $E$-equivalence class if $d_{T(E)}\left(0_{T}, P_{E}(\mu)\right)=\rho_{M}(0, \mu)$.

We defined a natural isomorphism mapping the tangent space to $T(E)$ at its basepoint onto a Banach space $A(E)^{*}$. That isomorphism is an isometry with respect to the infinitesimal Teichmüller metric on the tangent space and the usual norm on $A(E)^{*}$. Throughout this paper we will denote this infinitesimal Teichmüller norm by $\ell_{\mu} ;$ so $\ell_{\mu}$ is the norm of the linear functional

$$
\ell_{\mu}(\phi)=\iint_{\mathbf{C}} \mu \phi d x d y \quad \text { on } A(E)
$$

Henceforth, we will denote this by

$$
\left\|\ell_{\mu}\right\|_{T(E)}=\sup _{\|\phi\|=1}\left|\iint_{\mathbf{C}} \mu \phi d x d y\right|, \quad \phi \in A(E) .
$$

It is clear that $\left\|\ell_{\mu}\right\|_{T(E)} \leq\|\mu\|_{\infty}$ for $\mu$ in $L^{\infty}(\mathbf{C})$.

Definition 1.11. A Beltrami coefficient $\mu$ is infinitesimally extremal in its $E$ equivalence class, if $\left\|\ell_{\mu}\right\|_{T(E)}=\|\mu\|_{\infty}$.

The following proposition is obvious.

Proposition 1.12. If $E$ is a subset of $\widetilde{E}$ and $p_{\widetilde{E}, E}: T(\widetilde{E}) \rightarrow T(E)$ is the forgetful map, then

$$
d_{T(E)}\left(p_{\widetilde{E}, E}(s), p_{\widetilde{E}, E}(t)\right) \leq d_{T(\widetilde{E})}(s, t)
$$


for all $s$ and $t$ in $T(\widetilde{E})$.

When $\boldsymbol{E}$ is finite. Let $E$ be a finite set (as usual, 0,1 , and $\infty$ belong to $E)$. Its complement $E^{c}=\Omega$ is the Riemann sphere with punctures at the points of $E$. Since $T(E)$ and the classical Teichmüller space Teich $(\Omega)$ are quotients of $M(\mathbf{C})$ by the same equivalence relation, $T(E)$ can be naturally identified with $T e i c h(\Omega)$. It is given in Example 3.1 in [15]. For the reader's convenience, we include this discussion. Let $\theta: T(E) \rightarrow$ Teich $(\Omega)$ be the map defined by setting $\theta\left(P_{E}(\mu)\right)$ equal to the Teichmüller class of the restriction of $w^{\mu}$ to $\Omega$ (where, as usual, $\mu$ is in $M(\mathbf{C})$ ). It is clear that $\theta$ is a well-defined map of $T(E)$ into Teich $(\Omega)$. We claim that $\theta$ is injective. For, suppose that the restrictions of $w^{\mu}$ and $w^{\nu}$ to $\Omega$ are in the same Teichmüller class. Then, there is a conformal map $h$ of $w^{\mu}(\Omega)$ onto $w^{\nu}(\Omega)$ such that $\left(w^{\nu}\right)^{-1} \circ h \circ w^{\mu}$ is isotopic to the identity rel $E$. This map $h$ is the identity, for it is obviously a Möbius transformation and it fixes 0,1 , and $\infty$ because $w^{\mu}$ and $w^{\nu}$ are normalized. Therefore, $w^{\mu}$ and $w^{\nu}$ are $E$-equivalent, and so $\theta$ is injective. Also, $\theta$ is surjective, since the restriction map $\mu \mapsto \mu \mid \Omega$ from $M(\mathbf{C})$ to $M(\Omega)$ is bijective and $\theta\left(P_{E}(\mu)\right)=\Phi(\mu \mid \Omega)$ for all $\mu$ in $M(\mathbf{C})$, where $\Phi: M(\Omega) \rightarrow$ Teich $(\Omega)$ is the standard projection. This also shows that $\theta$ is biholomorphic, since $P_{E}$ and $\Phi$ induce the complex structures of $T(E)$ and Teich $(\Omega)$. Under this indentification $d_{T(E)}$ becomes the (classical) Teichmüller metric for Teich $(\Omega)$. Furthermore, the norm of $\ell_{\mu}$ is simply the norm of the linear functional that $\mu$ induces on the Banach space of integrable holomorphic functions on $\Omega$. For standard facts on classical Teichmüller spaces, the reader is refereed to the books $[9,11,19]$.

We need the following form of Teichmüller contraction for $T(E)$ when $E$ is a finite set. (Recall that when $E$ is finite, $T(E)$ is naturally identified with the classical Teichmüller space Teich $(\widehat{\mathbf{C}} \backslash E)$. )

Theorem 1.13. Let $\mu \in M(\mathbf{C})$, and $P_{E}(\mu)=\tau$ in $T(E)$. Let $\mu_{0}$ be an extremal in $P_{E}(\mu)$. Set $k_{0}=\left\|\mu_{0}\right\|_{\infty}, k=\|\mu\|_{\infty}, K_{0}=\left(1+k_{0}\right) /\left(1-k_{0}\right)$, and $K=(1+k) /(1-k)$. Then

$$
\frac{1}{K_{0}}-\frac{1}{K} \leq \frac{2}{1-k^{2}}\left(k-\|\ell\|_{T(E)}\right) \leq K-K_{0}
$$

See Theorem 2 in [5].

Remark 1.14. Earle proved this result for Teich $(X)$ where $X$ is any hyperbolic Riemann surface. He used the Reich-Strebel inequalities to obtain his result. We need the special case when $X=\widehat{\mathbf{C}} \backslash E$ and $E=\left\{0,1, \infty, \zeta_{1}, \cdots, \zeta_{n}\right\}, n \geq 1$.

Approximations by finite subsets. Let $E$ be infinite and let $E_{1}, E_{2}, \cdots, E_{n}, \cdots$ be a sequence of finite subsets of $E$ such that $\{0,1, \infty\} \subset E_{1} \subset E_{2} \subset \cdots \subset E_{n} \subset \cdots$ and $\bigcup_{n=1}^{\infty} E_{n}$ is dense in $E$. Let 0 be the basepoint of $T(E)$, and for each $n \geq 1$, let $\pi_{n}$ be the forgetful map $p_{E, E_{n}}$ from $T(E)$ to $T\left(E_{n}\right)$. For any $\tau$ in $T(E)$ and $n \geq 1$ let $\tau_{n}=\pi_{n}(\tau)$. In particular, $0_{n}=\pi_{n}(0)$ is the basepoint of $T\left(E_{n}\right)$ for all $n \geq 1$. By Proposition 1.12, we have

$$
d_{T\left(E_{n}\right)}\left(0_{n}, \tau_{n}\right) \leq d_{T\left(E_{n+1}\right)}\left(0_{n+1}, \tau_{n+1}\right) \leq d_{T(E)}(0, \tau)
$$

for all $\tau$ in $T(E)$ and $n \geq 1$.

The following two facts will be important in our paper. For proofs, see [15] and [16]. 
Proposition 1.15. For each $\tau$ in $T(E)$ the increasing sequence $\left\{d_{T\left(E_{n}\right)}\left(0_{n}, \tau_{n}\right)\right\}$ converges to $d_{T(E)}(0, \tau)$.

Proposition 1.16. Let the infinite closed set $E$ and the finite subsets $E_{n}, n \geq 1$, be as above, and let $\mu$ belong to $L^{\infty}(\mathbf{C})$. The sequence $\left\{\left\|\ell_{\mu}\right\|_{T\left(E_{n}\right)}\right\}$ is increasing and converges to $\left\|\ell_{\mu}\right\|_{T(E)}$.

We will also need the following theorem. This appears in Earle's paper [5].

Theorem 1.17. Let $V$ be a complex Banach space and $g: \Delta \rightarrow V$ be a holomorphic map with $g(0)=0$ and $\|g(t)\| \leq 1, \forall t \in \Delta$. Fix $t \in \Delta \backslash\{0\}$. If either of the inequalities $\left\|g^{\prime}(0)\right\| \leq 1$ or $\|g(t)\| \leq|t|$ is strict, then both are strict and

$$
\rho_{\Delta}\left(\frac{\|g(t)\|}{|t|},\left\|g^{\prime}(0)\right\|\right) \leq \rho_{\Delta}(0, t) .
$$

\section{Statements of the main results}

For classical Teichmüller spaces, the principle of Teichmüller contraction was proved in [8]. A sharp form of Teichmüller contraction was proved by Earle in [5]. Gardiner's result was extended to the generalized Teichmüller space $T(E)$ in [16], which proved a $\delta-\epsilon$ form of Teichmüller contraction. Our first result extends Earle's form of Teichmüller contraction to $T(E)$; this sharpens and improves the $\delta-\epsilon$ inequalities in [16].

Theorem I. Let $\mu \in M(\mathbf{C})$, and $P_{E}(\mu)=\tau$ in $T(E)$. Let $\mu_{0}$ be an extremal in the E-equivalence of $\mu$. Set $k_{0}=\left\|\mu_{0}\right\|_{\infty}, k=\|\mu\|_{\infty}, K_{0}=\left(1+k_{0}\right) /\left(1-k_{0}\right)$, and $K=(1+k) /(1-k)$. Then

$$
\frac{1}{K_{0}}-\frac{1}{K} \leq \frac{2}{1-k^{2}}\left(k-\|\ell\|_{T(E)}\right) \leq K-K_{0}
$$

Our next result is on holomorphic isometries from $\Delta$ into $T(E)$. This extends Theorem 5 in $[6]$ to $T(E)$.

Theorem II. Let $f: \Delta \rightarrow T(E)$ be holomorphic and let $t_{1} \in \Delta$. Suppose either that

(1) $d_{T(E)}\left(f\left(t_{1}\right), f\left(t_{2}\right)\right)=\rho_{\Delta}\left(t_{1}, t_{2}\right)$ for some $t_{2} \in \Delta \backslash\left\{t_{1}\right\}$,

or

(2) $F_{T(E)}\left(f\left(t_{1}\right), f^{\prime}\left(t_{1}\right)\right)=\frac{1}{1-\left|t_{1}\right|^{2}}$, then $f$ is a holomorphic isometry.

In [5], Earle proved a form of Schwarz's lemma for classical Teichmüller spaces. Our next result extends that theorem to $T(E)$. Let $f: \Delta \rightarrow T(E)$ be a basepoint preserving holomorphic map and set

$$
k_{0}(t)=\|\mu\|_{\infty} \text { if } t \in \Delta, f(t)=P_{E}(\mu) \text {, and } \mu \text { is extremal . }
$$

Theorem III. Let $f: \Delta \rightarrow T(E)$ be a basepoint preserving holomorphic map. Fix any $t$ in $\Delta \backslash\{0\}$. If either of the inequalities $\left\|f^{\prime}(0)\right\|_{T(E)} \leq 1$ or $k_{0}(t) \leq|t|$ is strict, then both are strict and

$$
\rho_{\Delta}\left(\frac{k_{0}(t)}{|t|},\left\|f^{\prime}(0)\right\|_{T(E)}\right) \leq 2 \rho_{\Delta}(0, t) .
$$

Our final theorem is on complex geodesics and unique extremality in $T(E)$. It extends Theorem 6 in $[6]$ to $T(E)$. We first need two definitions. 
Definition 2.1. A geodesic segment $J$ in $T(E)$ is the image of an injective continuous map $\alpha:[0,1] \rightarrow T(E)$ such that

$$
d_{T(E)}\left(\alpha\left(x_{0}\right), \alpha\left(x_{2}\right)\right)=d_{T(E)}\left(\alpha\left(x_{0}\right), \alpha\left(x_{1}\right)\right)+d_{T(E)}\left(\alpha\left(x_{1}\right), \alpha\left(x_{2}\right)\right)
$$

whenever $0 \leq x_{1} \leq x_{2} \leq x_{3} \leq 1$. The points $f(0)$ and $f(1)$ are called the endpoints of $J$. We say that the geodesic segment $J$ joins the points $\tau_{1}$ and $\tau_{2}$ in $T(E)$ if they are the endpoints of $J$.

Definition 2.2. A Beltrami coefficient $\mu$ in $M(\mathbf{C})$ is called uniquely extremal if $P_{E}(\nu) \neq P_{E}(\mu)$ whenever $\nu \in M(\mathbf{C}), \nu \neq \mu$, and $\|\nu\|_{\infty} \leq\|\mu\|_{\infty}$.

It is obvious that every "uniquely extremal" $\mu$ is extremal.

Theorem IV. Let $\mu_{0} \in M(\mathbf{C}), \mu_{0} \neq 0$ and $\mu_{0}$ be extremal in its E-equivalence class. Then the following four statements are equivalent:

(1) The Beltrami coefficient $\mu_{0}$ is uniquely extremal and $\left|\mu_{0}\right|=\left\|\mu_{0}\right\|_{\infty}$ a.e.

(2) There exists only one geodesic segment joining $P_{E}(0)$ and $P_{E}\left(\mu_{0}\right)$.

(3) There exists only one holomorphic isometry $f: \Delta \rightarrow T(E)$ such that $f(0)=$ $P_{E}(0)$ and $f\left(\left\|\mu_{0}\right\|_{\infty}\right)=P_{E}\left(\mu_{0}\right)$.

(4) There exists only one holomorphic map $g: \Delta \rightarrow M(\mathbf{C})$ such that $g(0)=0$ and $P_{E}\left(g\left(\left\|\mu_{0}\right\|_{\infty}\right)\right)=P_{E}\left(\mu_{0}\right)$.

Remark 2.3. Recall from $\S 1$ that when $E$ is finite, $T(E)$ is naturally identified with the classical Teichmüller space Teich $(\widehat{\mathbf{C}} \backslash E)$, and so, $T(E)$ is finite-dimensional. In that case all the above theorems are well-known; see [5], §7 and $\S 8$ in [6], and also $\S 9.3$ and $\S 9.5$ in [13]. Therefore, for the rest of our paper, the blanket assumption will be that $E$ is an infinite closed set and that 0,1 , and $\infty$ belong to $E$.

\section{Proof of Theorem I}

Let $\tau \in T(E), P_{E}\left(\mu=\tau\right.$, and $\mu_{0}$ be extremal in the $E$-equivalence class of $\mu$. So we have $P_{E}(\mu)=P_{E}\left(\mu_{0}\right)$ and $\left\|\mu_{0}\right\|_{\infty} \leq\|\mu\|_{\infty}$. Let $k=\|\mu\|_{\infty}$ and $k_{0}=\left\|\mu_{0}\right\|_{\infty}$. Also, let

$$
K=\frac{1+k}{1-k} \quad \text { and } \quad K_{0}=\frac{1+k_{0}}{1-k_{0}} .
$$

We follow the construction given immediately after Theorem 1.13 (in $\S 1$ ). Let $\tau_{n}=$ $\pi_{n}(\tau)$, and let $\mu_{0}(n)$ be extremal in its $E_{n}$-equivalence class. Let $k_{0}(n)=\left\|\mu_{0}(n)\right\|_{\infty}$ and let

$$
K_{0}(n)=\frac{1+k_{0}(n)}{1-k_{0}(n)}
$$

Since $T\left(E_{n}\right)$ is identified with the classical Teichmüller space $T e i c h\left(\widehat{\mathbf{C}} \backslash E_{n}\right)$, by Theorem 1.13, the following is true for all $n$ :

$$
\frac{1}{K_{0}(n)}-\frac{1}{K} \leq \frac{2}{1-k^{2}}\left(k-\left\|\ell_{\mu}\right\|_{T\left(E_{n}\right)}\right) \leq K-K_{0}(n) .
$$

Since $\mu_{0}(n)$ is extremal in its $E_{n}$-equivalence class, we have $d_{T\left(E_{n}\right)}\left(0_{n}, \tau_{n}\right)=\left\|\mu_{0}(n)\right\|_{\infty}$ $=k_{0}(n)$. Also, since $\mu_{0}$ is extremal in its $E$-equivalence class, we have $d_{T(E)}(0, \tau)=$ $\left\|\mu_{0}\right\|_{\infty}=k_{0}$. By Propositions 1.14 and 1.15, we have

$$
\lim _{n \rightarrow \infty} K_{0}(n)=K_{0} \quad \text { and } \quad \lim _{n \rightarrow \infty}\left\|\ell_{\mu}\right\|_{T\left(E_{n}\right)}=\left\|\ell_{\mu}\right\|_{T(E)} .
$$

Taking limits in Equation (3.1), we obtain

$$
\frac{1}{K_{0}}-\frac{1}{K} \leq \frac{2}{1-k^{2}}\left(k-\left\|\ell_{\mu}\right\|_{T(E)}\right) \leq K-K_{0} .
$$


The following two corollaries will be very useful in our paper.

Corollary 3.1. (Hamilton-Krushkal-Reich-Strebel extremality condition for $T(E)$ ) A Beltrami coefficient $\mu$ is extremal in its E-equivalence class if and only if it is infinitesimally extremal in its E-equivalence class.

The proof is obvious.

We follow the same notations as in Theorem I. Let $P_{E}(\mu)=\tau$, and let $\mu_{0}$ be extremal in its $E$-equivalence class. Let $k=\|\mu\|_{\infty}$ and $k_{0}=\left\|\mu_{0}\right\|_{\infty}$.

Corollary 3.2. If either $k_{0}$ or $\left\|\ell_{\mu}\right\|_{T(E)}$ is less than $k$ then both are less than $k$. Moreover,

$$
\rho_{\Delta}\left(\frac{k_{0}}{k}, \frac{\left\|\ell_{\mu}\right\|_{T(E)}}{k}\right) \leq \rho_{\Delta}(0, k) .
$$

The proof is straightforward. See, for example, the proof of Corollary 1 in [5].

\section{Proof of Theorem II}

Let $\mathcal{L}: L^{\infty}(\mathbf{C}) \rightarrow A(E)^{*}$ be the linear map that takes $\mu$ in $L^{\infty}(\mathbf{C})$ to the functional $\ell_{\mu}$ defined as

$$
\mathcal{L}(\mu)(\phi)=\ell_{\mu}(\phi)=\iint_{\mathbf{C}} \mu \phi d x d y, \quad \text { for } \phi \in A(E) .
$$

By Proposition 1.4, we can assume, without loss of generality that $t_{1}=0$ and that $f(0)=0$. We use the same notation 0 for the basepoints in $\Delta, M(\mathbf{C})$, and $T(E)$. By Proposition 1.6, there exists a holomorphic map $\widehat{f}: \Delta \rightarrow M(\mathbf{C})$ such that $\widehat{f}(0)=0$ and $P_{E} \circ \widehat{f}=f$.

Let us assume there is $t_{2} \in \Delta \backslash\{0\}$ such that $d_{T(E)}\left(0, f\left(t_{2}\right)\right)=\rho_{\Delta}\left(0, t_{2}\right)$. We have

$$
\rho_{\Delta}\left(0, t_{2}\right)=d_{T(E)}\left(0, f\left(t_{2}\right)\right) \leq \rho_{M}\left(0, \widehat{f}\left(t_{2}\right)\right) \leq \rho_{\Delta}\left(0, t_{2}\right)
$$

where $d_{T(E)}\left(0, f\left(t_{2}\right)\right) \leq \rho_{M}\left(0, \widehat{f}\left(t_{2}\right)\right)$ because $P_{E}: M(\mathbf{C}) \rightarrow T(E)$ is holomorphic, $P_{E}(0)=0$ and $P_{E}\left(\widehat{f}\left(t_{2}\right)\right)=f\left(t_{2}\right)$; and also, $\rho_{M}\left(0, \widehat{f}\left(t_{2}\right)\right) \leq \rho_{\Delta}\left(0, t_{2}\right)$ because $\widehat{f}: \Delta \rightarrow$ $M(\mathbf{C})$ is holomorphic and $\widehat{f}(0)=0$. From the above inequality, we get

$$
d_{T(E)}\left(0, f\left(t_{2}\right)\right)=\rho_{M}\left(0, \widehat{f}\left(t_{2}\right)\right)=\rho_{\Delta}\left(0, t_{2}\right) .
$$

Hence $\widehat{f}\left(t_{2}\right)$ is extremal and $\left\|\widehat{f}\left(t_{2}\right)\right\|_{\infty}=\left|t_{2}\right|$.

Let $g: \Delta \rightarrow A(E)^{*}$ be defined as $g=\mathcal{L} \circ \widehat{f}$; then $\|g(t)\| \leq\|\widehat{f}(t)\|_{\infty}<1$, for all $t$ in $\Delta$. For all $\mu \in L^{\infty}(\mathbf{C})$, we have $\left\|\ell_{\mu}\right\| \leq\|\mu\|_{\infty}$. We also have $g(0)=0$ since $\widehat{f}(0)=0$ and $\ell_{0}=0$. So we can apply Schwarz's Lemma to both $g$ and $\widehat{f}$, and since $\widehat{f}\left(t_{2}\right)$ is extremal, it will be infinitesimally extremal by Corollary 3.1. Hence we have

$$
\left\|g\left(t_{2}\right)\right\|=\left\|\ell_{\widehat{f}\left(t_{2}\right)}\right\|=\left\|\widehat{f}\left(t_{2}\right)\right\|_{\infty}=\left|t_{2}\right| .
$$

This is the case of equality in Schwarz's lemma, and hence we get

$$
\left\|g^{\prime}(0)\right\|=\left\|\widehat{f}^{\prime}(0)\right\|_{\infty}=1 \text {. }
$$

From the definition of $\mathcal{L}$ we see that $\mathcal{L}(\mu)=0$ if and only if $P_{E}^{\prime}(0) \mu=0$. Using chain rule we obtain

$$
\left\|\ell_{\mu}\right\|=\inf \left\{\|\nu\|_{\infty}: \ell_{\mu}=\ell_{\nu}\right\}=\inf \left\{\|\nu\|_{\infty}: P_{E}^{\prime}(0) \mu=P_{E}^{\prime}(0) \nu\right\} .
$$


Hence we get

$$
\left.\left\|\ell_{\widehat{f}^{\prime}(0)}\right\|=\inf \left\{\|\nu\|_{\infty}: P_{E}^{\prime}(0) \nu=P_{E}^{\prime}(0) \widehat{f}^{\prime}(0)\right\}=\inf \left\{\|\nu\|_{\infty}: P_{E}^{\prime}(0) \nu=f^{\prime}(0)\right)\right\},
$$

which gives

$$
1=\left\|\widehat{f}^{\prime}(0)\right\|_{\infty}=\left\|\ell_{\widehat{f}^{\prime}(0)}\right\|=F_{T(E)}\left(0, f^{\prime}(0)\right) .
$$

Since we assumed $t_{1}=0$ and $f(0)=0$, we obtain

$$
F_{T(E)}\left(f\left(t_{1}\right), f^{\prime}\left(t_{1}\right)\right)=\frac{1}{1-\left|t_{1}\right|^{2}} .
$$

This proves $1 \Rightarrow 2$.

Now let us assume 2 , that is there is a $t_{1} \in \Delta$ such that

$$
F_{T(E)}\left(f\left(t_{1}\right), f^{\prime}\left(t_{1}\right)\right)=\frac{1}{1-\left|t_{1}\right|^{2}} .
$$

Again without loss of generality we assume $t_{1}=0$ and $f(0)=0$. With our assumption we thus have $f: \Delta \rightarrow T(E)$ is a holomorphic map, and $f(0)=0$ and $F_{T(E)}\left(0, f^{\prime}(0)\right)=$ 1.

Consider the holomorphic map $\widehat{f}: \Delta \rightarrow M(\mathbf{C})$ such that $\widehat{f}(0)=0$ and $P_{E} \circ \widehat{f}=f$. Using Schwarz's lemma as before we observe that

$$
1=F_{T(E)}\left(0, f^{\prime}(0)\right) \leq\left\|\hat{f}^{\prime}(0)\right\|_{\infty} \leq 1 .
$$

This implies that $\left\|\widehat{f}^{\prime}(0)\right\|_{\infty}=1$.

Again let $g=\mathcal{L} \circ \widehat{f}$ that is $g(t)=\ell_{\widehat{f}(t)}$. We get $\left\|g^{\prime}(0)\right\|=\left\|\widehat{f}^{\prime}(0)\right\|_{\infty}=1$. This is the case of equality in Schwarz's lemma, and hence we get

$$
\|g(t)\|=\|\widehat{f}(t)\|_{\infty}=|t| \quad \text { for all } t \in \Delta .
$$

So for all $t$ in $\Delta, \widehat{f}(t)$ is extremal and $\|\widehat{f}(t)\|_{\infty}=|t|$. We see that for all $t$ in $\Delta$ the following is true because of extremality and the last equation:

$$
d_{T(E)}(0, f(t))=d_{T(E)}\left(P_{E}(0), P_{E}(\widehat{f}(t))\right)=\rho_{M}(0, \widehat{f}(t))=\rho_{\Delta}(0, t) .
$$

Since $t_{1}=0$ and $f(0)=0$ we get $d_{T(E)}(f(0), f(t))=\rho_{\Delta}(0, t)$, and so,

$$
d_{T(E)}\left(f\left(t_{1}\right), f(t)\right)=\rho_{\Delta}\left(t_{1}, t\right)
$$

for all $t$ in $\Delta$. So $2 \Rightarrow 1$ trivially, and actually does imply something stronger.

Finally, we will show that for all $t, t^{\prime}$ in $\Delta, d_{T(E)}\left(f(t), f\left(t^{\prime}\right)\right)=\rho_{\Delta}\left(t, t^{\prime}\right)$. If $t_{1}=t^{\prime}$ we have nothing to prove, so let us assume $t_{1} \neq t^{\prime}$. We have already seen that any $t \in \Delta$ could have been chosen as $t_{1}$ and hence we can simply assume $t=t_{1}$ and we thus get

$$
\rho_{\Delta}\left(t, t^{\prime}\right)=\rho_{\Delta}\left(t_{1}, t^{\prime}\right)=d_{T(E)}\left(f\left(t_{1}\right), f\left(t^{\prime}\right)\right)=d_{T(E)}\left(f(t), f\left(t^{\prime}\right)\right)
$$

which proves that $f$ is a holomorphic isometry.

We note the following corollary, whose proof is obvious.

Corollary 4.1. Let $f: \Delta \rightarrow T(E)$ be a holomorphic map with $f(0)=P_{E}(0)$. Let $t \in \Delta \backslash\{0\}$. Define $k_{0}(t)=\|\nu\|_{\infty}$ where $f(t)=P_{E}(\nu)$ and $\nu$ is extremal in its $E$ equivalence class. We also know that $\left\|f^{\prime}(0)\right\|_{T(E)}=F_{T(E)}\left(0, f^{\prime}(0)\right)$. Then $k_{0}(t)=|t|$ if and only if $\left\|f^{\prime}(0)\right\|_{T(E)}=1$. 


\section{Proof of Theorem III}

Let $f: \Delta \rightarrow T(E)$ be a baepoint preserving holomorphic map; by Proposition 1.6, there exists a holomorphic map $\widehat{f}: \Delta \rightarrow M(\mathbf{C})$, such that $\widehat{f}(0)=0$ and $f=P_{E} \circ \widehat{f}$. Let $V_{0}$ be the Banach space of all tangent vectors at the basepoint of $T(E)$. We also know that $P_{E}{ }^{\prime}(0)$ takes the tangent vectors $\nu$ in the tangent space at the basepoint of $M(\mathbf{C})$ (which is $L^{\infty}(\mathbf{C})$ ) to the functional $\ell_{\nu}$. So $P_{E}{ }^{\prime}(0) \equiv \mathcal{L}$. Let $g=\mathcal{L} \circ \widehat{f}$ such that $g(t)=\ell_{\widehat{f}(t)}$; then $g: \Delta \rightarrow V_{0}$ is holomorphic and

$$
f^{\prime}(0)=\left(P_{E} \circ \widehat{f}\right)^{\prime}(0)=P_{E}^{\prime}(0)\left(\widehat{f}^{\prime}(0)\right)=\mathcal{L}\left(\widehat{f}^{\prime}(0)\right)=\ell_{\widehat{f}^{\prime}(0)}=g^{\prime}(0)
$$

since $\mathcal{L}$ is linear. Let $t \in \Delta \backslash\{0\}$ be fixed and one of the following inequalities $\left\|f^{\prime}(0)\right\| \leq 1$ and $k_{0}(t) \leq|t|$ be strict, then both are strict by Corollary 4.1. So we get

$$
\left\|g^{\prime}(0)\right\|=\left\|f^{\prime}(0)\right\|<1
$$

and so by Theorem 1.16 we get $\|g(t)\|<|t|$ and hence $\left\|\ell_{\widehat{f}(t)}\right\|<|t|$ or $\left\|\ell_{\widehat{f}(t)}\right\|_{T(E)}<|t|$. By the same theorem we also get

$$
\rho_{\Delta}\left(\frac{\left\|\ell_{\widehat{f}(t)}\right\|_{T(E)}}{|t|},\left\|f^{\prime}(0)\right\|_{T(E)}\right) \leq \rho_{\Delta}(0, t) .
$$

If $\left\|\ell_{\widehat{f}(t)}\right\|_{T(E)}=\|\widehat{f}(t)\|_{\infty}$, then by Corollary 3.1, $\widehat{f}(t)$ is extremal and $k_{0}(t)=$ $\left\|\ell_{\widehat{f}(t)}\right\|_{T(E)}$ and so by (5.1) we get

$$
\rho_{\Delta}\left(\frac{k_{0}(t)}{|t|},\left\|f^{\prime}(0)\right\|_{T(E)}\right) \leq \rho_{\Delta}(0, t) .
$$

Suppose $\left\|\ell_{\widehat{f}(t)}\right\|_{T(E)}<\|\widehat{f}(t)\|_{\infty}$. Let $r=\frac{\|\widehat{f}(t)\|_{\infty}}{|t|}$. Let $k=\|\mu\|_{\infty}$ and $k_{0}=\left\|\mu_{0}\right\|_{\infty}$. By Corollary 3.2 we have

$$
\rho_{\Delta}\left(\frac{k_{0}}{k}, \frac{\left\|\ell_{\mu}\right\|_{T(E)}}{k}\right) \leq \rho_{\Delta}(0, k)
$$

So for $\mu=\widehat{f}(t), k=r|t|$ and $k_{0}(t)=k_{0}$, we have

$$
\rho_{\Delta}\left(\frac{k_{0}(t)}{r|t|}, \frac{\left\|\ell_{\widehat{f}(t)}\right\|_{T(E)}}{r|t|}\right) \leq \rho_{\Delta}(0, r|t|) .
$$

Let us consider the map $\alpha: \Delta \rightarrow \Delta$ given by $\alpha(z)=r z$; then $\alpha$ is holomorphic and $\alpha(0)=0$. Let

$$
\frac{k_{0}(t)}{r|t|}=a \quad \text { and } \quad \frac{\left\|\ell_{\widehat{f}(t)}\right\|_{T(E)}}{r|t|}=b .
$$

Then, $a, b \in \Delta$ and by Schwarz's lemma we get $\rho_{\Delta}(a r, b r) \leq \rho_{\Delta}(a, b)$. This gives

$$
\rho_{\Delta}\left(\frac{k_{0}(t)}{|t|}, \frac{\left\|\ell_{\widehat{f}(t)}\right\|_{T(E)}}{|t|}\right) \leq \rho_{\Delta}\left(\frac{k_{0}(t)}{r|t|}, \frac{\left\|\ell_{\widehat{f}(t)}\right\|_{T(E)}}{r|t|}\right)
$$

We also have

$$
\rho_{\Delta}(0, r|t|)=\rho_{\Delta}(\alpha(0), \alpha|t|) \leq \rho_{\Delta}(0,|t|)=\rho_{\Delta}(0, t) .
$$

Combining (5.4), (5.5), and (5.6), we get

$$
\rho_{\Delta}\left(\frac{k_{0}(t)}{|t|}, \frac{\left\|\ell_{\widehat{f}(t)}\right\|_{T(E)}}{|t|}\right) \leq \rho_{\Delta}(0, t) .
$$


Combining (5.1) and (5.7), and using the triangle inequality, we obtain

$$
\rho_{\Delta}\left(\frac{k_{0}(t)}{|t|},\left\|f^{\prime}(0)\right\|_{T(E)}\right) \leq 2 \rho_{\Delta}(0, t) .
$$

\section{Proof of Theorem IV}

Step 1. (2) implies (3). Let $f_{1}$ and $f_{2}$ be two holomorphic isometries from $\Delta$ into $T(E)$, such that $f_{1}(0)=f_{2}(0)=P_{E}(0)$ and $f_{1}\left(\left\|\mu_{0}\right\|_{\infty}\right)=f_{2}\left(\left\|\mu_{0}\right\|_{\infty}\right)=P_{E}\left(\mu_{0}\right)$. By (2) there is only one geodesic segment joining 0 and $P_{E}\left(\left\|\mu_{0}\right\|_{\infty}\right)$. Therefore, the image of the line segment $\left[0,\left\|\mu_{0}\right\|_{\infty}\right]$ is pointwise the same under both $f_{1}$ and $f_{2}$. This implies that the holomorphic mapping $f_{1}-f_{2}$ is identically zero on the line segment $\left[0,\left\|\mu_{0}\right\|_{\infty}\right]$, and so $f_{1}-f_{2}$ is identically zero on $\Delta$.

Step 2. (1) implies (4). Let $\mu_{0}$ be extremal and $\left|\mu_{0}\right|=\left\|\mu_{0}\right\|_{\infty}$ a.e. Let $g: \Delta \rightarrow$ $M(\mathbf{C})$ be a holomorphic map with $g(0)=0$ and $P_{E}\left(g\left(\left\|\mu_{0}\right\|_{\infty}\right)\right)=P_{E}\left(\left\|\mu_{0}\right\|_{\infty}\right)$. By Schwarz's lemma, $\left\|g\left(\left\|\mu_{0}\right\|_{\infty}\right)\right\|_{\infty} \leq\left\|\mu_{0}\right\|_{\infty}$. Since $\mu_{0}$ is uniquely extremal, we have $g\left(\left\|\mu_{0}\right\|_{\infty}\right)=\mu_{0}$. Consider a function $f$ in $\overline{M(\mathbf{C})}$ (the closure of $M(\mathbf{C})$ in $L^{\infty}(\mathbf{C})$ ), with $|f(z)|=1$ a.e. Let $h$ be another function in $\overline{M(\mathbf{C})}$ such that $h(z) \neq 0$ in $\mathbf{C} \backslash Z_{h}$ where $Z_{h}=\{z \in \mathbf{C}: h(z)=0\}$ and $m\left(Z_{h}\right)=0$, where $m$ denotes the usual Lebesgue measure. Let $E_{f}=\{z \in \mathbf{C}:|f(z)| \neq 1\}$. By our assumption, $m\left(E_{f}\right)=0$. Consider the function $f_{t}(z)=f(z)+t h(z)$. Let $F_{h}=\left\{f_{t}, t \in \bar{\Delta}\right\}$. Suppose $F_{h} \subset \overline{M(\mathbf{C})}$. For any $t \in \Delta$ define $H_{t}=\left\{z \in \mathbf{C}:\left|f_{t}(z)\right|>1\right\}$. Let $f(z)=e^{i \theta(z)}, h(z)=|h(z)| e^{i \phi(z)}$ and $l(z)=\phi(z)-\theta(z)$. Also, $t=|t| e^{i \psi}$. Then we have

$$
\left|f_{t}(z)\right|=\sqrt{1+|t|^{2}(|h(z)|)^{2}+2|t|(|h(z)|) \cos (l(z)+\psi)} .
$$

If $f_{t} \in \overline{M(\mathbf{C})}$, then $m\left(H_{t}\right)=0$, and if $z \in \mathbf{C} \backslash H_{t}$, then

$$
1+|t|^{2}(|h(z)|)^{2}+2|t|(|h(z)|) \cos (l(z)+\psi) \leq 1 .
$$

But $|t|^{2}(|h(z)|)^{2}+2|t|(|h(z)|) \cos (l(z)+\psi) \leq 0$. This implies

$$
-\cos l(z) \cos \psi-\sin l(z) \sin \psi \geq \frac{|t||h(z)|}{2} .
$$

Consider the functions $f_{1}, f_{i}, f_{-1}$ and $f_{-i}$. Let $G=E_{f} \cup Z_{h} \cup H_{1} \cup H_{i} \cup H_{-1} \cup H_{-i}$. By our assumption, $m(G)=0$ and if $z \in \mathbf{C} \backslash G$, then $h(z) \neq 0$, and

$$
-\cos l(z) \geq \frac{|h(z)|}{2}, \quad-\sin l(z) \geq \frac{|h(z)|}{2}, \quad \cos l(z) \geq \frac{|h(z)|}{2}, \quad \sin l(z) \geq \frac{|h(z)|}{2} .
$$

This is not possible. Therefore, at least one of the following functions $f_{1}, f_{-1}, f_{i}$, or $f_{-i}$ does not belong to $\overline{M(\mathbf{C})}$. This implies that $f$ is a complex extreme point of $\overline{M(\mathbf{C})}$. Let $\lambda=\frac{\mu_{0}}{\left\|\mu_{0}\right\|_{\infty}}$. Since $\left|\mu_{0}\right|=\left\|\mu_{0}\right\|_{\infty}$ a.e. we have $|\lambda|=1$ a.e. Therefore $\lambda$ is a complex extreme point for $\overline{M(\mathbf{C})}$.

Now define $h: \Delta \rightarrow M(\mathbf{C})$ as,

$$
h(t)= \begin{cases}\frac{g(t)}{t} & \text { if } t \neq 0, \\ g^{\prime}(0) & \text { if } t=0 .\end{cases}
$$

Then $h$ is holomorphic and $h\left(\left\|\mu_{0}\right\|_{\infty}\right)=\lambda$. By the strong maximum modulus principle (see Proposition 6.19 in [4]) we have $h(t)=\lambda$. This implies

$$
g(t)=t \lambda=\frac{t \mu_{0}}{\left\|\mu_{0}\right\|_{\infty}} .
$$

Since $\mu_{0}$ is uniquely extremal, $g$ is uniquely determined, and we are done. 
Step 3. (4) implies (3). Let $f: \Delta \rightarrow T(E)$ be a holomorphic isometry such that $f(0)=P_{E}(0)$ and $f\left(\left\|\mu_{0}\right\|_{\infty}\right)=P_{E}\left(\mu_{0}\right)$. Consider the holomorphic map $\widehat{f}: \Delta \rightarrow$ $M(\mathbf{C})$ such that $\widehat{f}(0)=0$ and $P_{E} \circ \widehat{f}=f$. Then $P_{E}\left(\widehat{f}\left(\left\|\mu_{0}\right\|_{\infty}\right)\right)=P_{E}\left(\mu_{0}\right)$. By the uniqueness condition in (4), we have

This implies

$$
\widehat{f}(t)=\frac{t \mu_{0}}{\left\|\mu_{0}\right\|_{\infty}}, \quad t \in \Delta .
$$

So $f$ is uniquely determined.

$$
f(t)=P_{E}\left(\frac{t \mu_{0}}{\left\|\mu_{0}\right\|_{\infty}}\right), \quad t \in \Delta .
$$

Step 4. (3) implies (1). We first show that if (3) holds, then $\left|\mu_{0}\right|=\left\|\mu_{0}\right\|_{\infty}$ a.e. Let $r \in(0,1)$ and $Z_{r}=\left\{z \in \mathbf{C}:\left|\mu_{0}(z)\right|<r\left\|\mu_{0}\right\|_{\infty}\right\}$ we need to show that $m\left(Z_{r}\right)=0$. Let $\chi_{r}$ be the characteristic function of $Z_{r}$. Let $\phi \in A(E)$, where $A(E)$ is the closed subspace of $L^{1}(\mathbf{C})$ consisting of maps holomorphic in $E^{c}$. Define functions $f_{1}: \Delta \rightarrow T(E)$ and $f_{r}: \Delta \rightarrow T(E)$ by

$$
f_{1}(t)=P_{E}\left(\frac{t \mu_{0}}{\left\|\mu_{0}\right\|_{\infty}}\right)
$$

and

$$
f_{r}(t)=P_{E}\left(\frac{t \mu_{0}}{\left\|\mu_{0}\right\|_{\infty}}+\frac{1-r}{2} t\left(t-\left\|\mu_{0}\right\|_{\infty}\left(\chi_{r} \frac{\mid \phi}{\phi}\right)\right)\right) .
$$

These maps are holomorphic and we also have $f_{1}(0)=f_{r}(0)=0$ and $f_{1}\left(\left\|\mu_{0}\right\|_{\infty}\right)=$ $f_{r}\left(\left\|\mu_{0}\right\|_{\infty}\right)=P_{E}\left(\mu_{0}\right)$. They are also isometries since $\rho_{\Delta}\left(0,\left\|\mu_{0}\right\|_{\infty}\right)=d_{T(E)}\left(0, P_{E}\left(\mu_{0}\right)\right)$. So, by (3) they coincide and we obtain

$$
0=f_{1}^{\prime}(0)-f_{r}^{\prime}(0)=\frac{1-r}{2}\left\|\mu_{0}\right\|_{\infty} P_{E}^{\prime}(0)\left(\chi_{r} \frac{|\phi|}{\phi}\right) .
$$

This implies

$$
P_{E}^{\prime}(0)\left(\chi_{r} \frac{|\phi|}{\phi}\right)=0
$$

Since $P_{E}^{\prime}(0)(\mu)=\ell_{\mu}$, we get

$$
\ell_{\left(\chi_{r} \frac{|\phi|}{\phi}\right)}=0
$$

In particular,

$$
\ell_{\left(\chi_{r} \frac{|\phi|}{\phi}\right)}(\phi)=0
$$

So,

$$
\iint_{Z_{r}}|\phi| d x d y=0
$$

This shows that $m\left(Z_{r}\right)=0$ since $\phi$ is an arbitrary function in $A(E)$. Let $Z=$ $\bigcup_{r \in \mathbf{Q} \cap(0,1)} Z_{r}$, then $m(Z)=0$. This shows that $\left|\mu_{0}\right|=\left\|\mu_{0}\right\|_{\infty}$ a.e. For any (normalized) quasiconformal homeomorphism $h$ of $\widehat{\mathbf{C}}$, we define its Beltrami coefficient as

$$
\mu_{h}=\frac{h_{\bar{z}}}{h_{z}}
$$

If $h$ and $j$ are two quasiconformal homeomorphisms, we have the composition formula

$$
\mu_{h \circ j}=\frac{\mu_{j}+\left(\mu_{h} \circ j\right) \alpha_{j}}{1+\bar{\mu}_{j}\left(\mu_{h} \circ j\right) \alpha_{j}}
$$


where

$$
\alpha_{j}=\frac{\left|j_{z}\right|^{2}}{\left(j_{z}\right)^{2}}
$$

If $\nu \in M(\mathbf{C})$, then by $w^{\nu}$ we mean the unique normalized quasiconformal homeomorphism with Beltrami coefficient $\nu$ a.e.

Let $\nu \in M(\mathbf{C})$ such that $\|\nu\|_{\infty} \leq\left\|\mu_{0}\right\|_{\infty}$ and $P_{E}(\nu)=P_{E}\left(\mu_{0}\right)$. Since $\mu_{0}$ is extremal, it follows that $\nu$ is also extremal and $\|\nu\|_{\infty}=\left\|\mu_{0}\right\|_{\infty}$. Hence $f(t)=$ $P_{E}\left(\frac{t \nu}{\|\nu\|_{\infty}}\right)$ is a holomorphic isometry. So, by (3) we obtain

$$
P_{E}\left(\frac{t \nu}{\|\nu\|_{\infty}}\right)=P_{E}\left(\frac{t \mu_{0}}{\left\|\mu_{0}\right\|_{\infty}}\right) .
$$

Since $\nu$ is extremal, by (3) we obtain $|\nu|=\|\nu\|_{\infty}=\left\|\mu_{0}\right\|_{\infty}$ a.e. Also, $P_{E}(s \nu)=$ $P_{E}\left(s \mu_{0}\right)$, for any $s$ in $(0,1)$.

So $\left(w^{s \mu_{0}}\right)^{-1} \circ w^{s \nu}$ is isotopic to the identity rel $E$. This implies $w^{\mu_{0}} \circ\left(w^{s \mu_{0}}\right)^{-1} \circ w^{s \nu}$ is isotopic to $w^{\mu_{0}} \mathrm{rel} E$. This implies $\left(w^{\mu_{0}}\right)^{-1} \circ w^{\lambda}$ is isotopic to the identity rel $E$, where

$$
w^{\lambda}=w^{\mu_{0}} \circ\left(w^{s \mu_{0}}\right)^{-1} \circ w^{s \nu} .
$$

This implies $P_{E}(\lambda)=P_{E}\left(\mu_{0}\right)$. Now let $h=w^{\mu_{0}} \circ\left(w^{s \mu_{0}}\right)^{-1}$ and $j=w^{s \mu_{0}}$ such that $h \circ j=w^{\mu_{0}}$. By the formula for composition of quasiconformal mappings, we get

$$
\left|\mu_{h} \circ j\right|=\frac{\left|\mu_{0}\right|(1-s)}{1-s\left|\mu_{0}\right|^{2}} .
$$

We know that $\left|\mu_{0}\right|=\left\|\mu_{0}\right\|_{\infty}$ a.e. Let $\left\|\mu_{0}\right\|_{\infty}=k$ and $s k=k^{\prime}$. We get

$$
\left|\mu_{h} \circ j\right|=\frac{k-k^{\prime}}{1-k k^{\prime}}=k^{\prime \prime} \text { a.e. }
$$

Since $j$ is quasiconformal and therefore absolutely continuous, it follows that $\left|\mu_{h}\right|=$ $k^{\prime \prime}$ a.e. Now let us consider $h=w^{\mu_{0}} \circ\left(w^{s \mu_{0}}\right)^{-1}$ and $j=w^{s \nu}$ so that $h \circ j=w^{\lambda}$. By similar calculations we obtain

$$
\lambda=\frac{s \nu+\left(\mu_{h} \circ j\right) \alpha_{j}}{1+s \bar{\nu}\left(\mu_{h} \circ j\right) \alpha_{j}} .
$$

Since $|s \nu|=k^{\prime}$ a.e. and $\left|\mu_{h} \circ j\right|=k^{\prime \prime}$ a.e. and $\left|\alpha_{j}\right|=1$ we write $s \nu=k^{\prime} e^{i \theta}$ a.e. and $\left(\mu_{h} \circ j\right) \alpha_{j}=k^{\prime \prime} e^{i \phi}$ a.e. Hence

$$
\lambda=e^{i \theta} \frac{k^{\prime}+k^{\prime \prime} e^{i l}}{1+k^{\prime} k^{\prime \prime} e^{i l}}
$$

where $l=\phi-\theta$. Therefore, $|\lambda|=\left|\frac{k^{\prime}+k^{\prime \prime} e^{i l}}{1+k^{\prime} k^{\prime \prime} e^{i l}}\right|$. Next, note that

$$
\begin{aligned}
\frac{k^{\prime}+k^{\prime \prime} e^{i l}}{1+k^{\prime} k^{\prime \prime} e^{i l}} \leq \frac{k^{\prime}+k^{\prime \prime}}{1+k^{\prime} k^{\prime \prime}} & \Longleftrightarrow \frac{\left(k^{\prime}+k^{\prime \prime} \cos l\right)^{2}+k^{\prime \prime 2} \sin ^{2} l}{\left(1+k^{\prime} k^{\prime \prime} \cos l\right)^{2}+k^{\prime 2} k^{\prime \prime 2} \sin ^{2} l} \leq \frac{k^{2}+2 k^{\prime} k^{\prime \prime}+k^{\prime \prime 2}}{1+2 k^{\prime} k^{\prime \prime}+k^{\prime 2} k^{\prime \prime 2}} \\
& \Longleftrightarrow\left(1-k^{\prime 2}\right)\left(1-k^{\prime \prime 2}\right)(1-\cos l) \geq 0 .
\end{aligned}
$$

The last inequality is true since $k^{\prime}<1, k^{\prime \prime}<1$ and $\cos l \leq 1$. So we get

$$
|\lambda| \leq \frac{k^{\prime}+k^{\prime \prime}}{1+k^{\prime} k^{\prime \prime}}=k
$$

Since $P_{E}\left(\mu_{0}\right)=P_{E}(\lambda)$ and $\mu_{0}$ is extremal, it follows that $\lambda$ is extremal. Hence $|\lambda|=k$ a.e. This implies

$$
\left|\frac{k^{\prime}+k^{\prime \prime} e^{i l}}{1+k^{\prime} k^{\prime \prime} e^{i l}}\right|=\frac{k^{\prime}+k^{\prime \prime} e^{i l}}{1+k^{\prime} k^{\prime \prime} e^{i l}}
$$


This implies

$$
\left(1-k^{2}\right)\left(1-k^{\prime \prime 2}\right)(1-\cos l)=0 .
$$

Since $k^{\prime}<1$ and $k^{\prime \prime}<1$, this holds if and only if $\cos l=1$, i.e. $\cos (\phi-\theta)=1$. This implies $s \nu=k^{\prime} e^{i \theta}$ a.e. and $\left(\mu_{h} \circ j\right) \alpha_{j}=k^{\prime \prime} e^{i \phi}$ a.e. have the same arguments and can be rewritten as $s \nu=k^{\prime} e^{i \theta}$ a.e. and $\left(\mu_{h} \circ j\right) \alpha_{j}=k^{\prime \prime} e^{i \theta}$ a.e.

We can write $\left(\mu_{h} \circ j\right) \alpha_{j}=m \cdot s \nu$ where $m=\frac{k^{\prime}}{k^{\prime \prime}}>0$, so

$$
\lambda=\nu \frac{s+m s}{1+m s^{2} k^{2}} .
$$

This shows that $\lambda$ is a positive multiple of $\nu$. Let us write (for simplicity) $\lambda=p \nu$ where $p>0$. So, $\|\lambda\|_{\infty}=p\|\nu\|_{\infty}$. But we have $\|\lambda\|_{\infty}=\|\nu\|_{\infty}=\left\|\mu_{0}\right\|_{\infty}=k>0$. So $p=1$ and hence $\lambda=\nu$ a.e. Hence

$$
w^{\nu}=w^{\lambda}=w^{\mu_{0}} \circ\left(w^{s \mu_{0}}\right)^{-1} \circ w^{s \nu} \text { a.e. } \Longrightarrow w^{\nu} \circ\left(w^{s \nu}\right)^{-1}=w^{\mu_{0}} \circ\left(w^{s \mu_{0}}\right)^{-1} \text { a.e. }
$$

Since $s \in(0,1)$ is arbitrary, letting $s \rightarrow 0$, we observe $w^{\nu}=w^{\mu_{0}}$ a.e and hence $\nu=\mu_{0}$ a.e. This proves that $\mu_{0}$ is uniquely extremal.

Step 5. (1) implies (2). Let $\mu_{0}$ be uniquely extremal, and $\left|\mu_{0}\right|=\left\|\mu_{0}\right\|_{\infty}=k$ a.e. Let $\alpha:[0,1] \rightarrow T(E)$ be an injective continuous map, defined by $\alpha(t)=P_{E}(t \mu)$, so that $\alpha([0,1])$ is a geodesic segment joining $P_{E}(0)$ and $P_{E}\left(\mu_{0}\right)$. We want to show this is the only geodesic segment joining $P_{E}(0)$ and $P_{E}\left(\mu_{0}\right)$.

Let us assume that there is another injective continuous map $\beta:[0,1] \rightarrow T(E)$, such that $\beta([0,1])$ is another geodesic segment joining $P_{E}(0)$ and $P_{E}\left(\mu_{0}\right)$. Let $\nu \in$ $M(\mathbf{C})$ be a point such that $P_{E}(\nu) \in \beta([0,1]) \backslash \alpha([0,1])$. Let $\nu_{0}$ be extremal in the E-equivalence class of $\nu$. Since $P_{E}\left(\nu_{0}\right)$ is an interior point of the geodesic segment we see that

$$
d_{T(E)}\left(P_{E}(0), P_{E}\left(\nu_{0}\right)\right) \leq d_{T(E)}\left(P_{E}(0), P_{E}\left(\mu_{0}\right)\right) .
$$

Since $\left|\mu_{0}\right|=k$ a.e. and $\nu_{0}$ is extremal, we see that $\left|\mu_{0}\right| \geq\left|\nu_{0}\right|$ a.e. Consider the mapping $w^{\eta}=w^{\mu_{0}} \circ\left(w^{\nu_{0}}\right)^{-1}$, so that $w^{\eta} \circ w^{\nu_{0}}=w^{\mu_{0}}$.

Let $\eta_{0}$ be the extremal in the E-equivalence class of $\eta$. Observe that $w^{\eta_{0}} \circ w^{\nu_{0}}=w^{\widetilde{\mu}}$ for some $\widetilde{\mu}$ such that $P_{E}(\widetilde{\mu})=P_{E}\left(\mu_{0}\right)$. So we get

and

$$
\left|\eta \circ w^{\nu_{0}}\right|=\left|\frac{\mu_{0}-\nu_{0}}{1-\overline{\nu_{0}} \mu_{0}}\right|
$$

$$
\left|\eta_{0} \circ w^{\nu_{0}}\right|=\left|\frac{\widetilde{\mu}-\nu_{0}}{1-\overline{\nu_{0}} \tilde{\mu}}\right|
$$

Let $\|\widetilde{\mu}\|_{\infty}=n$ and $\left\|\nu_{0}\right\|_{\infty}=l$. Since $\mu_{0}$ and $\nu_{0}$ are both extremal, we get $l<k \leq n$.

Now consider the map

$$
f(z)=\frac{z-a}{1-\bar{a} z}, \quad a \in \Delta .
$$

This map is holomorphic in $\Delta$ and $f(a)=0$. So, if $1>\delta_{1}>\delta_{2}>a$, then $a \in \overline{B_{\delta_{2}}(0)} \subset$ $B_{\delta_{1}}(0)$, where $B_{\delta}(0)=\{z \in \Delta:|z-a|<\delta\}$. Since $f$ is a Möbius transformation, by maximum modulus principle,

$$
\delta_{1}>\delta_{2} \Longleftrightarrow \sup _{z \in B_{\delta_{1}}(0)}|f(z)|=\sup _{z \in \partial B_{\delta_{1}}(0)}|f(z)|>\sup _{z \in \partial B_{\delta_{2}}(0)}|f(z)|=\sup _{z \in B_{\delta_{2}}(0)}|f(z)|
$$

and

$$
\delta_{1}=\delta_{2} \Longleftrightarrow \sup _{z \in B_{\delta_{1}}(0)}|f(z)|=\sup _{z \in \partial B_{\delta_{1}}(0)}|f(z)|=\sup _{z \in \partial B_{\delta_{2}}(0)}|f(z)|=\sup _{z \in B_{\delta_{2}}(0)}|f(z)| .
$$


Applying this to our problem we see that for all possible values of $\nu_{0}$, since $\|\widetilde{\mu}\|_{\infty}=n$ and $n \geq k$, we have

$$
\sup _{\widetilde{\mu}}\left|\frac{\widetilde{\mu}-\nu_{0}}{1-\overline{\nu_{0}} \widetilde{\mu}}\right| \geq \sup _{\mu_{0}}\left|\frac{\mu_{0}-\nu_{0}}{1-\overline{\nu_{0}} \mu_{0}}\right|
$$

So

$$
\sup _{\nu_{0}} \sup _{\widetilde{\mu}}\left|\frac{\widetilde{\mu}-\nu_{0}}{1-\overline{\nu_{0}} \widetilde{\mu}}\right| \geq \sup _{\nu_{0}} \sup _{\mu_{0}}\left|\frac{\mu_{0}-\nu_{0}}{1-\overline{\nu_{0}} \mu_{0}}\right| .
$$

This implies that $\left\|\eta_{0}\right\|_{\infty} \geq\|\eta\|_{\infty}$. Since $\left\|\eta_{0}\right\|_{\infty} \leq\|\eta\|_{\infty}$, we conclude that $\left\|\eta_{0}\right\|_{\infty}=\|\eta\|_{\infty}$. By the above discussion we have $n=k$, that is $\left\|\mu_{0}\right\|_{\infty}=\|\widetilde{\mu}\|_{\infty}$.

We conclude that $\widetilde{\mu}$ is extremal, and since $\mu_{0}$ is uniquely extremal, $\widetilde{\mu}=\mu_{0}$. So

$$
w^{\eta_{0}}=w^{\mu_{0}} \circ\left(w^{\nu_{0}}\right)^{-1} .
$$

This gives us

$$
d_{T(E)}\left(P_{E}(0), P_{E}\left(\eta_{0}\right)\right)=d_{T(E)}\left(P_{E}\left(\nu_{0}\right), P_{E}\left(\mu_{0}\right)\right) .
$$

Since $P_{E}(0), P_{E}\left(\nu_{0}\right)$ and $P_{E}\left(\mu_{0}\right)$ are on a geodesic segment, we have

$$
d_{T(E)}\left(P_{E}(0), P_{E}\left(\nu_{0}\right)\right)+d_{T(E)}\left(P_{E}\left(\nu_{0}\right), P_{E}\left(\mu_{0}\right)\right)=d_{T(E)}\left(P_{E}(0), P_{E}\left(\mu_{0}\right)\right) .
$$

Using Equation (6.2) we get

$$
d_{T(E)}\left(P_{E}(0), P_{E}\left(\nu_{0}\right)\right)+d_{T(E)}\left(P_{E}(0), P_{E}\left(\eta_{0}\right)\right)=d_{T(E)}\left(P_{E}(0), P_{E}\left(\mu_{0}\right)\right) .
$$

Since $\mu_{0}, \nu_{0}$, and $\eta_{0}$ are extremal in their respective equivalence classes, we get

$$
\rho_{\Delta}\left(0,\left\|\nu_{0}\right\|_{\infty}\right)+\rho_{\Delta}\left(0,\left\|\eta_{0}\right\|_{\infty}\right)=\rho_{\Delta}\left(0,\left\|\mu_{0}\right\|_{\infty}\right) .
$$

This implies

$$
\left\|\eta_{0}\right\|_{\infty}=\frac{\left\|\mu_{0}\right\|_{\infty}-\left\|\nu_{0}\right\|_{\infty}}{1-\left\|\nu_{0}\right\|_{\infty}\left\|\mu_{0}\right\|_{\infty}}
$$

Since we have

$$
w^{\eta_{0}}=w^{\mu_{0}} \circ\left(w^{\nu_{0}}\right)^{-1}
$$

we obtain

$$
\left|\eta_{0} \circ w^{\nu_{0}}\right|=\left|\frac{\mu_{0}-\nu_{0}}{1-\overline{\nu_{0}} \mu_{0}}\right| .
$$

Let $\nu_{0}=s \mu_{0}, s=|s| e^{i \phi}$ and $\mu_{0}=k e^{i \theta}$ and $|s|<1$. By Equation (6.4) we get

$$
\left\|\eta_{0}\right\|_{\infty}=k \frac{1-\sup |s|}{1-\sup |s| k^{2}}
$$

By Equation (6.5) we get

$$
\left|\eta_{0} \circ w^{\nu_{0}}\right|=k \mid \frac{1-|s| e^{i(\phi-\theta)}}{1-|s| k^{2} e^{i(\theta-\phi)} \mid} .
$$

Setting $\omega=\phi-\theta$, we rewrite this as

$$
\left|\eta_{0} \circ w^{\nu_{0}}\right|=k\left|\frac{1-|s| e^{i \omega}}{1-|s| k^{2} e^{-i \omega}}\right| .
$$


It is easy to see that

$$
\begin{aligned}
& \left|\frac{1-|s| e^{i \omega}}{1-|s| k^{2} e^{-i \omega}}\right| \geq \frac{1-|s|}{1-|s| k^{2}} \\
& \quad \Longleftrightarrow \frac{(1-|s| \cos \omega)^{2}+|s|^{2} \sin ^{2} \omega}{\left(1-|s| k^{2} \cos \omega\right)^{2}+|s|^{2} k^{4} \sin ^{2} \omega} \geq \frac{(1-|s|)^{2}}{\left(1-|s| k^{2}\right)^{2}} \\
& \quad \Longleftrightarrow\left(1-k^{2}\right)\left(1-|s|^{2} k^{2}\right)(1-\cos \omega) \geq 0 .
\end{aligned}
$$

The last inequality is true since $k<1, s<1$ and $\cos \omega \leq 1$. So Equation (6.7) gives

$$
\left|\eta_{0} \circ w^{\nu_{0}}\right| \geq k \frac{1-|s|}{1-|s| k^{2}}
$$

Hence

$$
\left\|\eta_{0}\right\|_{\infty} \geq k \frac{1-|s|}{1-|s| k^{2}}
$$

It is easy to see from Equations (6.6) and (6.9) that

$$
k \frac{1-\sup |s|}{1-\sup |s| k^{2}} \geq k \frac{1-|s|}{1-|s| k^{2}} \Longrightarrow|s|=\sup |s|:=S .
$$

From Equations (6.6) and (6.8) we get

$$
k \frac{1-S}{1-S k^{2}}=\left\|\eta_{0}\right\|_{\infty} \geq\left|\eta_{0} \circ w^{\nu_{0}}\right|=k\left|\frac{1-S e^{i \omega}}{1-S k^{2} e^{i \omega}}\right| \geq k \frac{1-S}{1-S k^{2}} .
$$

This gives

This is true if and only if

$$
k\left|\frac{1-S e^{i \omega}}{1-S k^{2} e^{i \omega}}\right|=k \frac{1-S}{1-S k^{2}} .
$$

$$
\left(1-k^{2}\right)\left(1-S^{2} k^{2}\right)(1-\cos \omega)=0 .
$$

That can happen only when $\cos \omega=\cos (\phi-\theta)=1$, which means $\nu_{0}$ and $\mu_{0}$ have the same arguments and hence we can write $\nu_{0}=S \mu_{0}, 1>S>0$. But that contradicts our assumption. So we conclude that there is only one geodesic segment joining $P_{E}(0)$ and $P_{E}\left(\mu_{0}\right)$, which completes the proof.

Acknowledgement. I want to thank the referee for her/his careful reading and valuable suggestions. I also want to thank Professor Yunping Jiang who read an earlier draft of this paper and gave several helpful comments.

\section{References}

[1] Ahlfors, L. V.: Lectures on quasiconformal mappings. Second edition. - Univ. Lecture Ser. 38, Amer. Math. Soc., 2006.

[2] Ahlfors, L. V., and L. Bers: Riemann's mapping theorem for variable metrics. - Ann. of Math. 72, 1960, 385-404.

[3] Chae, S. B.: Holomorphy and calculus in normed spaces. - Marcel Dekker, New York, 1985.

[4] Dineen, S.: The Schwarz lemma. - Oxford Math. Monogr., Oxford Univ. Press, Oxford, 1990.

[5] Earle, C. J.: Schwarz's lemma and Teichmüller contraction. - Contemp. Math. 311, 2002, 79-85.

[6] Earle, C. J., I. Kra, and S. L. Krushkal: Holomorphic motions and Teichmüller spaces. Trans. Amer. Math. Soc. 343:2, 1994, 927-948. 
[7] Earle, C. J., and S. Mitra: Variation of moduli under holomorphic motions. - Contemp. Math. 256, 2000, 39-67.

[8] Gardiner, F. P.: - On Teichmüller contraction. - Proc. Amer. Math. Soc. 118, 1993, 865-875.

[9] Gardiner, F.P., and N. LAkic: Quasiconformal Teichmüller theory. - Math. Surveys Monogr. 76, Amer. Math. Soc., Providence, 1993.

[10] Harris, L. A.: Schwarz-Pick systems of pseudometrics for domains in normed linear spaces. - In: Advances in holomorphy, North-Holland Math. Studies 34, North-Holland, Amsterdam, 1979, 345-406.

[11] HubBard, J. H.: Teichmüller theory and applications to geometry, topology and dynamics. Volume I. Teichmüller theory. - Matrix Editions, Ithaca, NY, 2006.

[12] Jiang, Y., and S. Mitra: Douady-Earle section, holomorphic motions, and some applications. - Contemp. Math. 575, 2012, 219-251.

[13] Lehto, O.: Univalent functions and Teichmüller spaces. - Grad. Texts in Math. 109, SpringerVerlag, Berlin, 1987.

[14] LiEB, G.S.: Holomorphic motions and Teichmüller space. - Ph.D. dissertation, Cornell University, 1990.

[15] Mitra, S.: Teichmüller spaces and holomorphic motions. - J. Anal. Math. 81, 2000, 1-33.

[16] Mitra, S.: Teichmüller contraction in the Teichmüller space of a closed set in the sphere. Israel J. Math. 125, 2001, 45-51.

[17] Mitra, S.: On extensions of holomorphic motions - a survey. - In: Geometry of Riemann surfaces, edited by Gardiner, González-Diez and Kourouniotis, London Math. Soc. Lecture Note Ser. 368, 2010, 283-308.

[18] Mitra, S., and H. Shiga: Extensions of holomorphic motions and holomorphic families of Möbius groups. - Osaka J. Math. 47, 2010, 1167-1187.

[19] NaG, S.: The complex analytic theory of Teichmüller spaces. - John Wiley and Sons, New York, 1988.

[20] Rudin, W.: Functional analysis. Second edition. - McGraw-Hill, New York, 1992.

[21] Stodkowski, Z.: Holomorphic motions and polynomial hulls. - Proc. Amer. Math. Soc. 111, $1991,347-355$.

Received 4 October 2016 • Accepted 23 December 2016 